\title{
In vitro and in vivo mechanism of immunomodulatory and antiviral activity of Edible Bird's Nest (EBN) against influenza A virus (IAV) infection
}

\begin{abstract}
Ethnopharmacological relevance: For centuries, Edible Bird Nest (EBN) has been used in treatment of variety of respiratory diseases such as flu and cough as a Chinese natural medicine. Aim of the study: This natural remedy showed the potential to inhibit influenza A virus (IAV). However, little is known about the mechanism of this process and also the evaluation of this product in an animal model. Hence, the current study was designed to elucidate the antiviral and immunomodulatory effects of EBN against IAV strain A/Puerto Rico/8/1934 (H1N1). Materials and methods: First, influenza infected MDCK cells treated with EBNs from two locations of Malaysia (Teluk Intan and Gua Madai) that prepared with different enzymatic preparations were analyzed by RT-qPCR and ELISA for detection of viral and cytokines genes. The sialic acid composition of these EBNs was evaluated by $\mathrm{H}-$ NMR. Subsequently, after toxicity evaluation of EBN from Teluk Intan, antiviral and immunomodulatory effects of this natural product was evaluated in BALB/c mice by analysis of the viral NA gene and cytokine expressions in the first week of the infection. Results: EBN showed high neuraminidase inhibitory properties in both in vitro and in vivo, which was as effective as Oseltamivir phosphate. In addition, EBN decreased NS1 copy number $(p<0.05)$ of the virus along with high immunomodulatory effects against IAV. Some of the immune changes during treatment of IAV with EBN included significant increase in IFN $\gamma, \mathrm{TNF} \alpha$, $\mathrm{NF} \kappa \mathrm{B}$, IL2, some proinflammatory cytokines like IL1 $\beta$, IL6, and cytokines with regulatory properties like IL10, IL27, IL12, CCL2 and IL4 depends on the stage of the infection. EBNs from two locations contained different composition of sialic acid and thymol derivatives, which gave them different antiviral properties. EBN from Gua Madai that contained more acetylated sialic acid (Neu2,4,7,8,9 Ac6) showed higher antiviral activity. Conclusion: The findings of this study support the antiviral activity of EBN against influenza virus and validate the traditional usage of this natural remedy by elucidation of toxicity and the molecular mechanism of action.
\end{abstract}

Keyword: Edible Bird Nest (EBN); Influenza A virus; Cytokine; Immunomodulatory; Antiviral 